\title{
Correction to: MoGrr1, a novel F-box protein, is involved in conidiogenesis and cell wall integrity and is critical for the full virulence of Magnaporthe oryzae
}

\author{
Min Guo ${ }^{1} \cdot$ Fei Gao ${ }^{1} \cdot$ Xiaolei Zhu ${ }^{1} \cdot$ Xiang Nie $^{1} \cdot$ YueMin Pan $^{1} \cdot$ Zhimou Gao $^{1}$ \\ Published online: 17 October 2018 \\ (C) Springer-Verlag GmbH Germany, part of Springer Nature 2018
}

\section{Correction to: Appl Microbiol Biotechnol (2015) 99:8075-8088 \\ https://doi.org/10.1007/s00253-015-6820-x}

The authors of Guo et al. (2015) would like to correct image errors in their article (https://doi.org/10.1007/ s00253-015-6820-x). Two images were incorrectly edited in Fig. 6 and Fig. 7. In Fig. 6, images were mistakenly used for strains Mogrr1-7 and Mogrrl-com in panel a, and for Mogrr1-com in panel d, respectively. We replaced them with original ones as showed below. In Fig. 7, panel (a), the image of Mogrr1-com was incorrect. The correct Fig. 7 is shown below. We confirm that these changes do not alter any findings of this work.

\section{Reference}

Guo M, Gao F, Zhu X, Nie X, Pan Y, Gao Z (2015) MoGrr1, a novel Fbox protein, is involved in conidiogenesis and cell wall integrity and is critical for the full virulence of Magnaporthe oryzae. Applied Microbiology and Biotechnology 99:8075-8088
The online version of the original article can be found at https://doi.org/ $10.1007 / \mathrm{s} 00253-015-6820-\mathrm{x}$

Min Guo

kandylemon@163.com

Zhimou Gao

gaozhimou@126.com

1 Department of Plant Pathology, College of Plant Protection, Anhui Agricultural University, Hefei 230036, China 
Fig. 6 Mogrrl is essential for appressorium development in M. oryzae. A. Appressorium development at hyphal tips. Bars $=40 \mu \mathrm{m}$. B. Statistical analysis of the appressorium formation rate. More than 100 hyphal tips were observed each time. C. The appressorium deformity rate was measured and statistically analyzed. D. Appressorium formation on the surface of rice plant leaves. Bars $=30 \mu \mathrm{m}$. E. Measurement of appressoria formed on rice plant leaves. Asterisks in Fig. 6b, c and e indicate significant differences from the control $(P<0.01)$. ">10 lesions" in Fig. 6e indicates that more than 10 lesions were measured for each strain. The experiment was repeated three times with three cultures each and representative results from one experiment are shown a

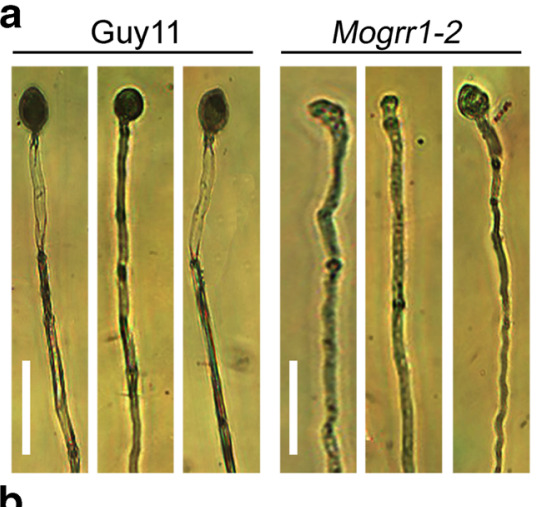

b

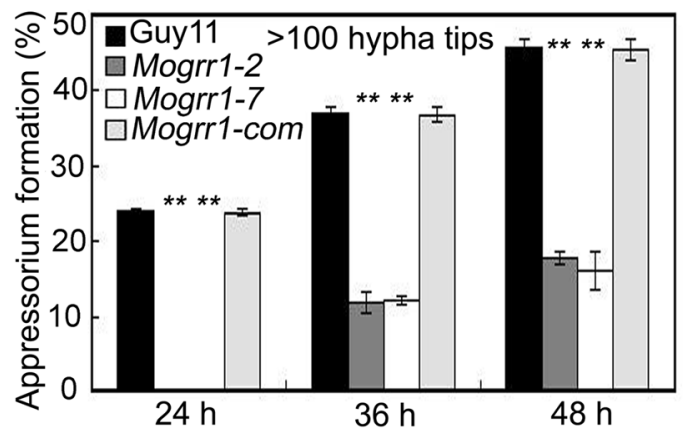

d
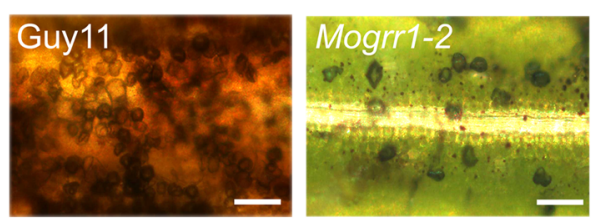

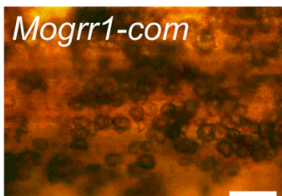

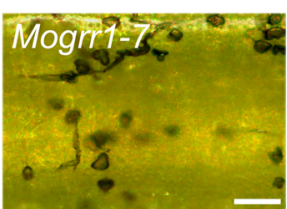

e

C
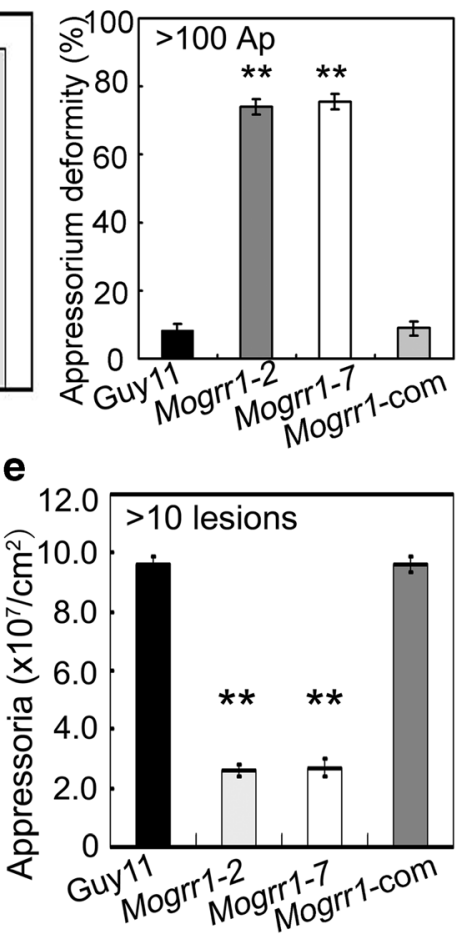


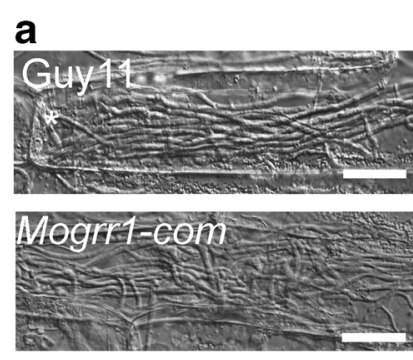

C

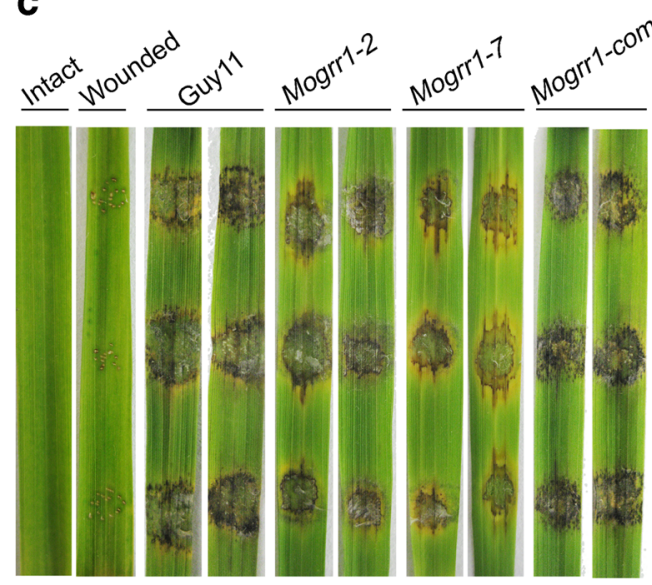

Fig. 7 Mogrrl deletion results in impaired appressorium penetration and attenuated virulence. A. Barley leaf penetration assay. Asterisks indicate appressoria. Bars $=20 \mu \mathrm{m}$. B. Statistical analysis of appressorium-mediated penetration. The appressoria that penetrated into barley epidermal cells were counted, and the data were statistically analyzed. C. Pathogenicity assay on abraded rice plant leaves. The tested strains were incubated on wounded rice leaves, and their virulence was evaluated at 5 days after incubation. D. b

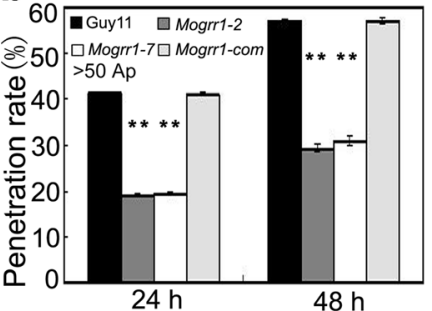

d

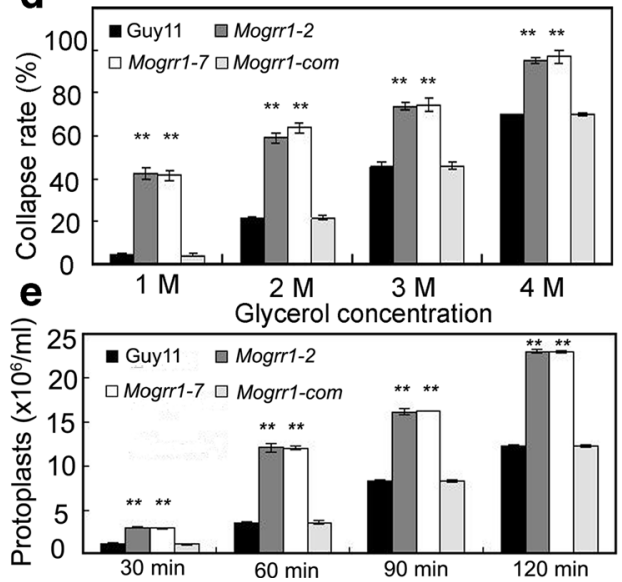

Measurement of collapsed appressoria. At least 100 appressoria were observed at each glycerol concentration. E. Protoplasts were released under the treatment of lysing enzymes. Asterisks in Fig. $7 \mathrm{~b}, \mathrm{~d}$ and e indicate significant differences to the control $(P<0.01)$. " $>50 \mathrm{AP} "$ in Fig. $7 \mathrm{~b}$ indicates that more than 50 appressoria were measured for each strain. Similar results were obtained in three repeated experiments, and the representative data from one experiment are shown 